\title{
Plasma nitrite/nitrate levels: a new biomarker for pulmonary arterial hypertension?
}

\author{
James R. Klinger \\ Affiliation: Division of Pulmonary, Sleep and Critical Care Medicine, Rhode Island Hospital, and Warren Alpert \\ Medical School, Brown University, Providence, RI, USA. \\ Correspondence: James R. Klinger, Division of Pulmonary, Sleep and Critical Care Medicine, Rhode Island \\ Hospital, 593 Eddy Street, Providence, RI 02903, USA. E-mail: james_klingerlabrown.edu
}

@ERSpublications

Plasma nitrate/nitrite levels are lower in patients with pulmonary hypertension and correlate with disease severity http://ow.ly/JzYD304gWv4

The role of endothelial-derived nitric oxide (NO) and its downstream signalling pathways in modulating vascular tone and remodelling has been one of the most intriguing areas of vascular biology over the last quarter of a century. Its identification as the endothelial-derived relaxing factor responsible for relaxation of vascular smooth muscle led to the designation of NO as the "molecule of the year" by the journal Science in 1992 [1], and to a Nobel Prize in Physiology or Medicine for L.J. Ignarro, R.F. Furchgott and F. Murad in 1998. It is now well appreciated that the vascular endothelium, in response to a variety of stimuli, synthesises NO from L-arginine by activation of endothelial-derived NO synthase (eNOS), which then diffuses to adjacent vascular smooth muscle cells and activates soluble guanylate cyclase (sGC) leading to synthesis of cyclic guanosine monophosphate (cGMP). The increase in cytoplasmic levels of this secondary messenger activates a variety of mechanisms that decrease intercellular calcium and dephosphorylate the myosin light chain. The overall result is a potent mechanism by which the endothelium can induce vascular smooth muscle relaxation [2].

Initial interest in the role of $\mathrm{NO}$ in the pulmonary circulation focused on this ability to reverse pulmonary vasoconstriction. However, further study revealed that $\mathrm{NO}$ signalling also has antiproliferative and anti-inflammatory properties, and important modulatory effects on apoptosis, angiogenesis and endothelin synthesis that play vital roles in pulmonary vascular remodelling as well $[3,4]$. Sentinel studies have shown that NO blunts acute hypoxic pulmonary vasoconstriction in isolated rat lungs and human volunteers [5, 6], whereas mice with targeted gene deletion of eNOS have elevated pulmonary artery pressure (PAP) and increased muscularisation of pulmonary arteries in response to chronic hypoxia [7, 8]. However, inhalation of NO or increasing endogenous NO signalling via a variety of methods has been shown to lower PAP, and reduce right ventricular hypertrophy and muscularisation of pulmonary arteries in animal models of pulmonary hypertension [9-11]. These studies led to the development of a variety of therapeutic agents that target the NO/cGMP pathway for the treatment of pulmonary arterial hypertension (PAH). These agents include phosphodiesterase type-5 (PDE5) inhibitors that delay the degradation of cGMP [12], sGC stimulators that both stimulate sGC and enhance NO-induced activation of sGC [13], and inhalation of NO itself [14].

Considering the salient effects of enhanced NO/cGMP signalling on attenuating pulmonary hypertensive responses, it is no wonder that many investigators have suspected that deficiency in NO signalling may contribute to the pathogenesis of PAH. A considerable body of evidence has accrued to support this hypothesis, including the following abnormalities found in patients with $\mathrm{PAH}$ : 1) decreased expression of eNOS in endothelial cells that line pre-capillary pulmonary arteriole resistance vessels [15], 2) lower levels of the substrates and co-factors needed for eNOS-mediated generation of NO such as L-arginine (the

Received: Aug 022016 | Accepted: Aug 222016

Conflict of interest: Disclosures can be found alongside this article at erj.ersjournals.com

Copyright @ERS 2016 
primary substrate for eNOS) and tetrahydrobiopterin [16, 17], 3) higher levels of arginase that degrades L-arginine [18], and 4) higher plasma levels of asymmetric dimethyl arginine that competitively inhibits the binding of L-arginine to NO synthase [19]. Together, these findings suggest that patients with PAH have impaired ability to generate NO in the pulmonary circulation. Indeed, the concentration of NO in exhaled air is lower in patients with idiopathic pulmonary arterial hypertension (IPAH) and PAH associated with connective tissue disease than in healthy controls [20, 21].

This strong association between NO signalling and $\mathrm{PAH}$ makes it intriguing to consider whether some assessment of NO signalling could be used to assess the severity of pulmonary vascular disease and predict outcome in patients with PAH. Unfortunately, none of the indices of NO synthesis described above is readily attainable or has been found to be amenable to development into a convenient, reproducible measure that could be used as a biomarker. Another approach then may be to examine changes in $\mathrm{NO}$ metabolism. Most synthesised $\mathrm{NO}$ is metabolised by the progressive oxidation of $\mathrm{NO}$ to nitrite $\left(\mathrm{NO}_{2}^{-}\right)$and nitrate $\left(\mathrm{NO}_{3}^{-}\right)$. Circulating nitrite/nitrate $\left(\mathrm{NO}_{x}\right)$ levels not only reflect the level of $\mathrm{NO}$ synthesis but also act as a reservoir of alternative substrate for $\mathrm{NO}$ synthesis. NO metabolites can be chemically reduced to NO in vivo by haem proteins, such as xanthine oxidoreductase and aldehyde oxidase, that exhibit nitrite reductase activity [22]. Thus, measurement of circulating $\mathrm{NO}_{x}$ could serve as an index of vascular $\mathrm{NO}$ synthesis and/or reflect the ability of a patient to synthesise NO via this alternative pathway.

In this issue of European Respiratory Journal (ERJ), ZHANG et al. [23] examine the association between plasma $\mathrm{NO}_{x}$ levels and severity of $\mathrm{PH}$ and clinical outcome in patients with $\mathrm{PAH}$. They measured plasma $\mathrm{NO}_{x}$ levels in 104 patients with $\mathrm{PAH}$ at the time of first right heart catheterisation and in 110 healthy controls, and followed them prospectively for a mean of $26 \pm 9$ months. They found that circulating $\mathrm{NO}_{x}$ levels in patients with $\mathrm{PAH}$ were only about a third of the level found in controls. Furthermore, plasma $\mathrm{NO}_{x}$ levels were lower in 18 of the $104 \mathrm{PAH}$ with a bone morphogenic protein receptor-2 (BMPR2) mutation than in the 85 patients without. Plasma $\mathrm{NO}_{x}$ levels correlated inversely with mean pulmonary artery pressure (mPAP) and pulmonary vascular resistance (PVR), and directly with cardiac output and cardiac index. More importantly, PAH patients with lower circulating $\mathrm{NO}_{x}$ levels had higher 1- and 3-year mortality rates. In fact, during the 3-year follow-up period, patients with a plasma $\mathrm{NO}_{x}$ concentration of $<10 \mu \mathrm{mol} \cdot \mathrm{L}^{-1}$ had a mortality rate that was nearly four times higher than those with levels $>10 \mu \mathrm{mol} \cdot \mathrm{L}^{-1}$ (37.5\% versus $10.4 \%$ ). Interestingly, plasma $\mathrm{NO}_{x}$ level had greater ability to predict death than mPAP, PVR, cardiac output and plasma brain natriuretic peptide levels by area under the curve in receiver operating characteristic analysis. The negative prognostic value of low plasma $\mathrm{NO}_{x}$ persisted after controlling for additional factors including age, sex, BMPR2 mutation, World Health Organization functional class and 6-min walk distance.

These findings add to a growing body of data that suggests that impaired NO synthesis either contributes to the pathogenesis of $\mathrm{PAH}$ or is a marker of endothelial dysfunction that reflects $\mathrm{PAH}$ severity and disease progression. More importantly, this study identifies a potentially new biomarker that may contribute to the diagnosis and management of $\mathrm{PAH}$. It is particularly interesting to note that plasma $\mathrm{NO}_{x}$ levels were lower in PAH patients with BMPR2 mutations. These patients had higher mPAP and PVR than those without BMPR2 mutation, and their lower plasma $\mathrm{NO}_{x}$ may reflect more severe disease. However, BMPR2 mutations have also been associated with impaired NO synthesis [24] and it is possible that this reduction in $\mathrm{NO}$ synthesis contributed to more advanced disease.

Plasma $\mathrm{NO}_{x}$ was measured by a commercially available assay in venous blood collected in standard EDTA-containing tubes with $0.005 \%$ butylated hydroxytoluene to improve stability. Samples were frozen for up to a month at $-80^{\circ} \mathrm{C}$ with little deterioration and acceptable inter-assay variation, suggesting that plasma $\mathrm{NO}_{x}$ levels are amenable to routine collection, storage and measurement.

Limitations of the study include its single-centre design and restriction of study subjects to those with IPAH. Patients with IPAH and a variety of comorbidities that could affect plasma $\mathrm{NO}_{x}$ levels were also excluded, including those with systemic hypertension, hyperlipidaemia or type 2 diabetes mellitus, and patients who used tobacco, excessive alcohol or nonsteroidal anti-inflammatory drugs. These comorbidities are not uncommon in patients with IPAH and it remains to be determined if plasma nitrates have similar predictive value for disease severity or outcome in these patients. Further studies will also be needed to examine the prognostic value of plasma $\mathrm{NO}_{x}$ levels in other types of PAH. For example, children with $\mathrm{PAH}$ associated with congenital left-to-right shunt have been found to have higher $\mathrm{NO}_{x}$ levels than controls [25]. This may be due to increased pulmonary vascular endothelial NO synthesis caused by increased pulmonary blood flow. Other limitations to the study include the lack of follow-up $\mathrm{NO}_{x}$ levels to determine if changes in plasma $\mathrm{NO}_{x}$ reflect disease progression and the omission of the investigators to examine whether baseline $\mathrm{NO}_{x}$ levels were able to predict clinical response to PAH-specific medications. The latter point is particularly important in that the rapid development of numerous pharmacological therapies for $\mathrm{PAH}$ and the absence of direct comparator trials make it difficult to determine which 
patients are best suited for each therapy. It would have been intriguing to see if baseline plasma $\mathrm{NO}_{x}$ is useful in determining which patients are more likely to respond to medications that target the NO/cGMP pathway, such as PDE5 inhibitors or sGC stimulators. It would also be of great interest to see if any of the currently available $\mathrm{PAH}$ medications are capable of increasing plasma $\mathrm{NO}_{x}$ levels and if the increase correlates with patient outcome.

Finally, the conversion of inorganic nitrates to NO is one of the mechanisms by which diets that are rich in nitrate-containing vegetables can enhance endothelial function in cardiovascular disease. Previous studies in mice have shown that hypoxic pulmonary hypertension can be attenuated by treating drinking water with $\mathrm{NO}_{2}^{-}$or $\mathrm{NO}_{3}^{-}$[26]. In these studies, dietary $\mathrm{NO}_{3}^{-}$increased plasma and lung concentrations of $\mathrm{NO}_{3}^{-}$and cGMP, and these increases mirrored the reduction in right ventricular hypertrophy and pulmonary vascular remodelling. Taken together, these studies and the report by ZHANG et al. [23] in this issue of the ERJ suggest that plasma nitrates may offer a potential biomarker that can be used to assess disease severity, help determine prognosis and perhaps provide us with a valuable tool to assess which patients are most likely to benefit from enhancing NO signalling and monitoring their response to treatment.

\section{References}

$1 \quad$ Koshland Jr DE. The molecule of the year. Science 1992; 258: 1861.

2 Surks HK. cGMP-dependent protein kinase I and smooth muscle relaxation: a tale of two isoforms. Circ Res 2007; 101: 1078-1080.

3 Hampl V, Herget J. Role of nitric oxide in the pathogenesis of chronic pulmonary hypertension. Physiol Rev 2000; 80: $1337-1372$.

4 Klinger JR, Abman SH, Gladwin MT. Nitric oxide deficiency and endothelial dysfunction in pulmonary arterial hypertension. Am J Respir Crit Care Med 2013; 188:639-646.

5 Rich GF, Roos CM, Anderson SM, et al. Inhaled nitric oxide: dose response and the effects of blood in the isolated rat lung. J Appl Physiol 1993; 75: 1278-1284.

6 Frostell CG, Blomqvist H, Hedenstierna G, et al. Inhaled nitric oxide selectively reverses human hypoxic pulmonary vasoconstriction without causing systemic vasodilation. Anesthesiology 1993; 78: 427-435.

7 Steudel W, Scherrer-Crosbie M, Bloch KD, et al. Sustained pulmonary hypertension and right ventricular hypertrophy after chronic hypoxia in mice with congenital deficiency of nitric oxide synthase 3. J Clin Invest 1998; 101: 2468-2477.

8 Fagan KA, Fouty BW, Tyler RC, et al. The pulmonary circulation of homozygous or heterozygous eNOS-null mice is hyperresponsive to mild hypoxia. J Clin Invest 1999; 103: 291-299.

9 Kouyoumdjian C, Adnot S, Levame M, et al. Continuous inhalation of nitric oxide protects against development of pulmonary hypertension in chronically hypoxic rats. J Clin Invest 1994; 94: 578-584.

10 Budts W, Pokreisz P, Nong Z, et al. Aerosol gene transfer with inducible nitric oxide synthase reduces hypoxic pulmonary hypertension and pulmonary vascular remodeling in rats. Circulation 2000; 102: 2880-2885.

11 Campbell AI, Kuliszewski MA, Stewart DJ. Cell-based gene transfer to the pulmonary vasculature: endothelial nitric oxide synthase overexpression inhibits monocrotaline-induced pulmonary hypertension. Am J Respir Cell Mol Biol 1999; 21: 567-575.

12 Galiè N, Ghofrani HA, Torbicki A, et al. Sildenafil citrate therapy for pulmonary arterial hypertension. $N$ Engl J Med 2005; 353: 2148-2157.

13 Ghofrani HA, Galiè N, Grimminger F, et al. PATENT-1 Study Group. Riociguat for the treatment of pulmonary arterial hypertension. N Engl J Med 2013; 369: 330-340.

14 Muller W, Kachel W, Lasch P, et al. Inhaled nitric oxide for avoidance of extracorporeal membrane oxygenation in the treatment of severe persistent pulmonary hypertension of the newborn. Intensive Care Med 1996; 22: 71-76.

15 Giaid A, Saleh D. Reduced expression of endothelial nitric oxide synthase in the lungs of patients with pulmonary hypertension. N Engl J Med 1995; 333: 214-221.

16 Morris CR, Kato GJ, Poljakovic M, et al. Dysregulated arginine metabolism, hemolysis-associated pulmonary hypertension, and mortality in sickle cell disease. JAMA 2005; 294: 81-90.

17 Davydova MP, Postnikov AB, D'iakonov KB, et al. Involvement of tetrahydrobiopterin in local change of endothelium-dependent vasorelaxation in pulmonary hypertension. Ross Fiziol Zh Im I M Sechenova 2003; 89: $1516-1522$.

$18 \mathrm{Xu}$ W, Kaneko FT, Zheng S, et al. Increased arginase II and decreased NO synthesis in endothelial cells of patients with pulmonary arterial hypertension. FASEB J 2004; 18: 1746-1748.

19 Pullamsetti S, Kiss L, Ghofrani HA, et al. Increased levels and reduced catabolism of asymmetric and symmetric dimethylarginines in pulmonary hypertension. FASEB J 2005; 19: 1175-1177.

20 Kaneko FT, Arroliga AC, Dweik RA, et al. Biochemical reaction products of nitric oxide as quantitative markers of primary pulmonary hypertension. Am J Respir Crit Care Med 1998; 158: 917-923.

21 Kharitonov SA, Cailes JB, Black CM, et al. Decreased nitric oxide in the exhaled air of patients with systemic sclerosis with pulmonary hypertension. Thorax 1997; 52: 1051-1055.

22 Bueno M, Wang J, Mora AL, et al. Nitrite signaling in pulmonary hypertension: mechanisms of bioactivation, signaling, and therapeutics. Antioxid Redox Signal 2013; 18: 1797-1809.

23 Zhang R, Wang X-J, Zhang H-D, et al. Profiling nitric oxide metabolites in patients with idiopathic pulmonary arterial hypertension. Eur Respir J 2016; 48: 1386-1395.

24 Gangopahyay A, Oran M, Bauer EM, et al. Bone morphogenetic protein receptor II is a novel mediator of endothelial nitric-oxide synthase activation. J Biol Chem 2011; 286: 33134-33140.

25 Takaya J, Teraguchi M, Nogi S, et al. Relation between plasma nitrate and mean 20 pulmonary arterial pressure in ventricular septal defect. Arch Dis Child 1998; 79: 498-501.

26 Baliga RS, Milsom AB, Ghosh SM, et al. Dietary nitrate ameliorates pulmonary hypertension: cytoprotective role for endothelial nitric oxide synthase and xanthine oxidoreductase. Circulation 2012; 125: 2922-2932. 